\title{
Agronomic
} Journal jê

\section{ANÁLISE MORFOMÉTRICA DA BACIA HIDROGRÁFICA DO RIO DOS PATOS, GO}

\section{MORPHOMETRIC ANALYSIS OF THE HYDROGRAPHIC BASIN OF RIO DOS PATOS, GO}

\author{
Laiane Cristina Freitas Pereira ${ }^{1}$, Gustavo Henrique Mendes Brito ${ }^{2 *}$, Igor Leonardo Vespucci 3 , Ivandro \\ José de Freitas Rocha ${ }^{4}$
}

${ }^{1}$ Bacharel em Agronomia pela Faculdade Evangélica de Goianésia, laiane_crvg@hotmail.com

2 Docente, Mestre em Engenharia Agrícola da Faculdade Evangélica de Goianésia,gh.mendes.brito@gmail.com

${ }^{3}$ Bacharel em Agronomia, Doutorando em Agronegócio, Programa de Pós Graduação em Agronegócio da Universidade Federal de Goiás (UFG), igorvespucci@agronomo.eng.br

${ }^{4}$ Docente, Bacharel em Informática e Mestrando em Engenharia agrícola pelo Programa de Pós Graduação em Engenharia Agrícola da Universidade Estadual de Goiás (UEG), ivandro_rocha@yahoo.com.br

\section{Info}

Recebido: 08/2018

Publicado: 03/2019

ISSN: 2595-6906

\section{Palavras-Chave \\ rede de drenagem, geoprocessamento, sistema de informação geográfica. \\ Keywords: \\ drainage network, geoprocessing, geographic information system.}

\begin{abstract}
A bacia hidrográfica é formada por uma região de drenagem de um rio e seus afluentes, suas características geomorfológicas influenciam no comportamento hidrológico e ambiental do local que está localizada, sendo associadas a parâmetros físicos obtidos pela caracterização morfométrica de bacias hidrográficas. A caracterização morfométrica expressa a relação solo-superfície de uma região hidrográfica, leva em consideração o relevo, rede de drenagem e processos ambientais que permitem analisar as características geomorfológicas da região. Com base no exposto o objetivo com este trabalho foi realizar a caracterização morfométrica da bacia hidrográfica do Rio dos Patos, Goiás, utilizando a base cartográfica do estado de Goiás na escala 1:250.000, sendo calculado o coeficiente de compacidade, fator de forma, densidade de drenagem,
\end{abstract} índice de circularidade, declividade média da bacia, área e perímetro de drenagem, e a ordem dos cursos d'água, utilizando o sistema de informação geográficas (SIG) como auxilio do software ArcGis. A bacia hidrográfica do Rio dos Patos possui formato alongado e não susceptível a enchentes em condições normais de precipitações.

\section{Resumo}

The hydrographic basin is formed by a drainage region of a river and its tributaries, its geomorphological characteristics influence the hydrological and environmental behavior of the site that is located, being associated with physical parameters obtained by the morphometric characterization of hydrographic basins. The morphometric characterization expressed to the soil-surface relation of a hydrographic region, takes into account the relief, drainage network and environmental processes that allow to analyze the geomorphological characteristics of the region. Based on the above, the objective of this work was to perform the morphometric characterization of the Rio dos Patos basin, Goiás, using the cartographic base of the state of Goiás in the scale 1: 250000, being calculated the coefficient of compactness, shape factor, density drainage area, circularity index, mean slope of the basin, area and perimeter of drainage, and the order of watercourses, using the geographic information system (GIS) as an aid to ArcGis software. The water catchment area of the Rio dos Patos is elongated and not susceptible to flooding under normal rainfall conditions. 


\section{INTRODUÇÃO}

As bacias hidrográficas são definidas como regiões de drenagens de rios principais e seus afluentes, formam uma área de captação natural da água precipitada, que em contato com as águas subterrâneas, converge para um único ponto de saída, o exutório (MOTA et al., 2017). Elas passam por um processo físico de formação, onde a forma e o relevo do local podem provocar sedimentação e influenciar a quantidade de água produzida (CARDOSO et al., 2006; TONELLO et al., 2006). Com isso, as características geomorfológicas e o tipo da cobertura vegetal influenciam no comportamento hidrológico de uma bacia hidrográfica, ou seja, na ocorrência, distribuição e movimentação da água, por isso se torna necessário informações como área, relevo, solo, drenagem, entre outras, obtidas a partir de mapas, fotografias aéreas, imagens de satélite, levantamentos topográficos, entre outras técnicas que podem facilitar o estudo da dinâmica ambiental destes locais (LIMA et al, 1986; TUCCI et al., 2003).

Essas informações são conhecidas como parâmetros morfométrico, e estão relacionados com estudos de parâmetros físicos de uma bacia hidrográfica devido aos fenômenos hidrológicos ou ambientais em que ela é submetida. A partir do estudo morfométrico é possível conhecer o formato da bacia, indicar características geométricas, da rede de drenagem e de relevo (GARBRECHT et al, 1999; GOBBI et al, 2008).

Para tais estudos, tem-se utilizado modelos digitais de elevação (MDE) e as técnicas de geoprocessamento no mapeamento, delimitação e representações geomorfológicas das bacias hidrográficas (MOTA et al., 2017). Os MDEs consistem na representação matemática e computacional da distribuição espacial de dados altimétricos na superfície terrestre, e vêm sendo utilizados em diversos estudos relacionados aos recursos hídricos, sejam no delineamento de redes de drenagem, na determinação dos limites de bacias hidrográficas, nos cálculos de declividade, altitudes, entre outros (TORRES et al., 2006; TEIXEIRA et al., 2018),

Ou seja, a partir da caracterização morfométrica de uma bacia hidrográfica pode-se tomar conhecimento sobre elaboração de um plano de ação para a mesma, levantando problemas que nela residem, identificando conflitos para poder buscar soluções e recomendações para recuperar o meio ambiente, de forma que possa organizar uma gestão integral dos recursos naturais, permitindo a execução de manejos para uma boa preservação, principalmente da água (BENTES-GAMA, 2010).

Com base no exposto, o objetivo com este trabalho foi realizar a caracterização morfométrica da bacia hidrográfica do Rio dos Patos de forma a entendermos o comportamento da bacia de drenagem.

\section{MATERIAL E MÉTODOS}

\section{CARACTERIZAÇÃO DA ÁREA EM ESTUDO}

O estudo foi realizado na bacia hidrográfica Rio dos Patos, Go, sua nascente é no município de Pirenópolis, que deságua no Rio Maranhão, com latitudes $14^{\circ} 50^{\prime} 30^{\prime \prime} \mathrm{S}$ e longitudes $48^{\circ} 40^{\prime} 00^{\prime \prime} \mathrm{W}$ 


\section{(Figura 1).. Está inserida na área de drenagem do temperaturas médias variam de $22^{\circ}$ a $25^{\circ} \mathrm{C}$, o clima Alto Tocantins até a confluência do Rio Preto. da região é tropical úmido (BRASIL e Banha a região de Goianésia-Go, apresentando CARVALHO 1998).} precipitação pluvial média de $1.600 \mathrm{~mm}$ anuais, as

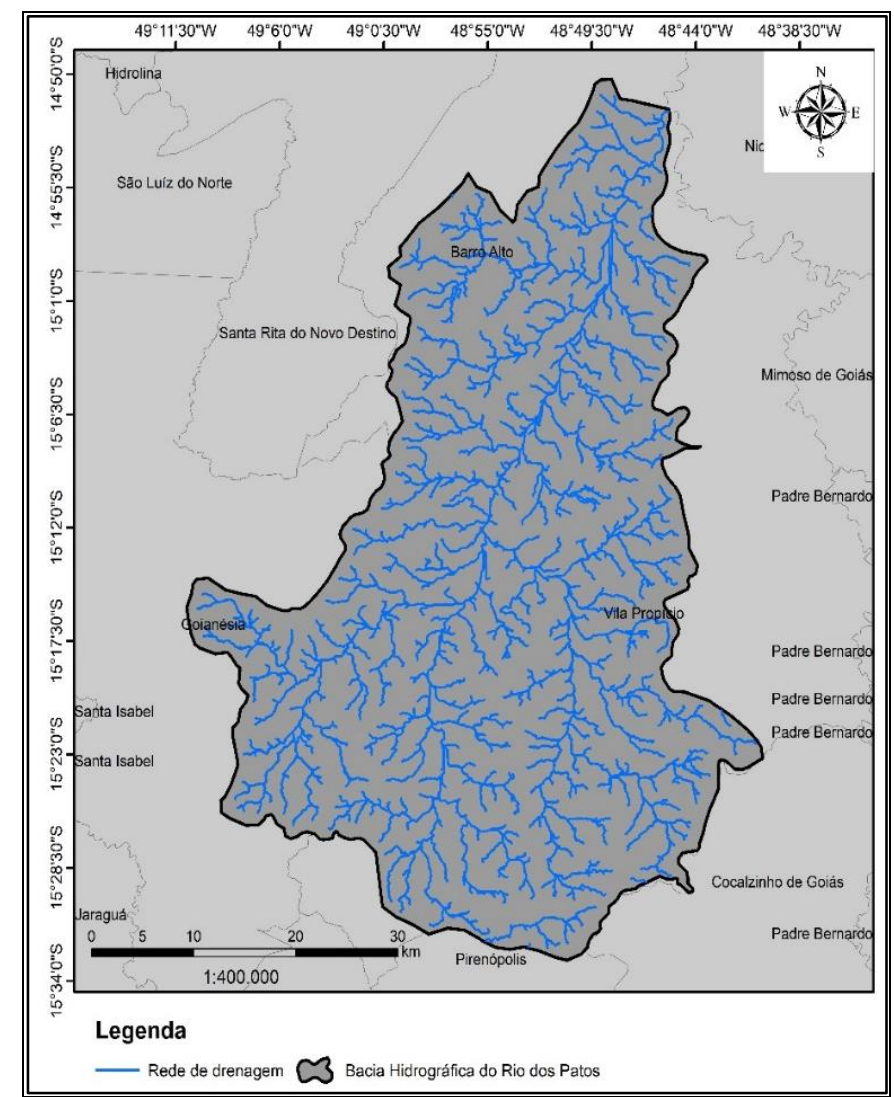

Figura 1. Mapa de localização da bacia hidrográfica do Rio Dos Patos, GO.

\section{CARACTERIZAÇÃO MORFOMÉTRICA DA BACIA HIDROGRÁFICA}

A delimitação da área da bacia hidrográfica e características físicas foram realizadas a partir da aquisição de imagens SRTM (Shutle Radar Topography Mission) com 30 metros de resolução espacial e auxílio dp software de sistemas de informações geográficas ArcMap 10.2. As variáveis geométricas analisadas foram: índice de compacidade $(\mathrm{Kc})$; fator de forma $(\mathrm{F})$; índice de circularidade (ke); densidade da drenagem (Dd); área da bacia (A); perímetro (P); declividade média (D); ordem dos cursos d'água, conforme a metodologia proposta por Cardoso et al (2006).

\section{COEFICIENTE DE COMPACIDADE}

O coeficiente de compacidade $(\mathrm{Kc})$ estabelece a relação entre o perímetro da bacia e a circunferência de um círculo de área igual à da bacia, ou seja, é a relação da forma da bacia com um círculo, ambos em $\mathrm{km}$. Quanto mais irregular a bacia for, será maior o $(\mathrm{Kc})$, pois quanto mais o coeficiente se aproximar de 1,0, maior será chances de enchentes da bacia. $\mathrm{O}$ valor adimensional desse coeficiente será de acordo com a forma da bacia. Um índice mínimo $(1,0)$ do coeficiente representará uma bacia circular, e uma bacia alongada o valor é superior a 1,0. Sendo assim, quando o Kc de uma bacia se aproximar da 
unidade sua propensão a cheias será maior (VILLELA; MATTOS; 1975). O índice de compacidade foi calculado por essa fórmula, sendo que: Kc é o coeficiente de compacidade (adimensional), P é o perímetro (m) e A é a área de drenagem $\left(\mathrm{m}^{2}\right)$.

$$
K_{C}=0,28 \frac{P}{\sqrt{A}}
$$

\section{FATOR DE FORMA}

O fator de forma é definido pelo tempo de concentração da água na bacia hidrográfica após uma precipitação, sendo que quanto maior a concentração, menor a chance de vazão de enchente (CARDOSO et al, 2006). A chance de uma inundação será maior quando o fator de forma for mais próximo de $(1,0)$ ou seja, mais próximo da forma circular (CHRISTOFOLETTI, 1980). A fórmula para calcular o fator de forma é a seguinte, sendo que F é o fator de forma (adimensional), A é a área de drenagem (m2) e L é o comprimento do eixo da bacia (m).

$$
F=\frac{A}{L^{2}}
$$

\section{ÍNDICE DE CIRCULARIDADE}

O índice de circularidade é relacionado entre a área da bacia e a área do círculo do mesmo parâmetro, ambos $\mathrm{em} \mathrm{km}^{2}$, com o valor máximo de 1,0. Quando o valor do índice de circularidade for maior e se aproximar de $(1,0)$ a bacia terá maior suscetibilidade a enchentes, já que esse valor corresponde à forma circular. E se o valor se distanciar a mesma terá formato alongado, com chances menores às cheias (CHRISTOFOLETTI 1980). A fórmula para calcular é a seguinte, sendo que Ke é o índice de circularidade, e A é a área de drenagem (m2) e P é o perímetro (m).

$$
K e=\frac{A \times 4 \pi}{p^{2}}
$$

\section{DENSIDADE DA DRENAGEM}

A densidade de drenagem vai indicar maior ou menor velocidade com que a água deixa a bacia. Vai correlacionar o comprimento dos canais de escoamento com a área da bacia de drenagem, ou seja, vai dizer o quanto a bacia é eficiente em sua drenagem, relacionando os canais de escoamento e área total da bacia (HORTON 1945). A equação é a seguinte, sendo que: Dd é a densidade de drenagem em $\mathrm{km} / \mathrm{km} 2$, Lt é o comprimento total de todos os canais em km, e A é a Área da bacia em $\mathrm{km} 2$.

$$
D d=\frac{L_{t}}{A}
$$

\section{ÁREA E PERÍMETRO DA BACIA}

A área e o perímetro da bacia foram calculados a partir das coordenadas UTM, Fuso 22S e auxílio do ArcMap 10.1 com a função Calculate Geometry. A área da bacia hidrográfica é um conjunto do sistema fluvial, de plano horizontal e possui toda área drenada (CHRISTOFOLETTTI 1980). O comprimento da linha imaginária ao longo do curso d'água é o que determina o perímetro da bacia hidrográfica (SMITH 1950).

\section{ORDEM DOS CURSOS D’ÁGUA}

As ordens dos cursos são classificadas em canais, onde uma linha de água sem tributários é considerada $1^{a}$ ordem. Os de $2^{a}$ ordem são quando duas linhas de água da primeira ordem se juntam, formando um rio com afluentes de $1^{\mathrm{a}}$ ordem, e assim sucessivamente, gerando canais de $3^{\mathrm{a}}, 4^{\mathrm{a}}$ ordem (SILVEIRA, 2001). 


\section{RESULTADOS E DISCUSSÕES}

Para caracterização da bacia utilizou-se

parâmetros morfométricos, apresentados na

Tabela 1.

Tabela 1. Resultados da caracterização morfometrica da bacia hidrográfica do Rio dos Patos, Go.

\begin{tabular}{lc}
\hline Parâmetros morfométricos & Resultados \\
\hline Área de drenagem $(\mathrm{A})\left(\mathrm{km}^{2}\right)$ & 2280,69 \\
Perímetro $(\mathrm{P})(\mathrm{km})$ & 274,42 \\
Comprimento do eixo da bacia $(\mathrm{L})(\mathrm{km})$ & 101,36 \\
Comprimento total dos leitos $(\mathrm{Lt})(\mathrm{km})$ & 1769,97 \\
Coeficiente de compacidade $(\mathrm{Ke})$ & 1,61 \\
Fator de Forma (F) & 0,22 \\
Densidade da drenagem (Dd) & 0,78 \\
Índice de circularidade (IC) & 0,38 \\
Ordem da bacia & $6^{\text {a Ordem }}$ \\
\hline
\end{tabular}

Possivelmente a bacia hidrográfica em estudo possui características que a tornam pouco susceptível a enchentes e inundações em condições normais de precipitações. Corroborando com os resultados Cardoso et al., (2006) que definem que os principais fatores associados ao risco de inundações são o coeficiente de compacidade próximo a 1,0 e o tempo que a água ficará concentrada na bacia hidrográfica após uma precipitação, sendo este tempo definido pelo fator de forma.

A área deste estudo apresentou dimensões semelhantes com Alves et al., (2016) que também realizaram caracterização morfométrica em uma bacia hidrográfica, com coeficiente de compacidade $(1,553)$, fator de forma $(0,23)$, índice de circularidade $(0,41)$, caracterizando a bacia do Ribeirão das Abóboras em Rio Verde - GO, como pouco susceptível a enchentes.
Reis et al., (2017) ao caracterizarem a bacia do Rio Guaxindiba - RJ, também identificaram forma alongada na sua área de estudo devido apresentar variáveis com valores com coeficiente de compacidade igual a 2,7542 , fator de forma de 0,2123 e índice de circularidade de 0,1299, por isso a bacia foi classificada como menos suscetível.

Na bacia hidrográfica de Igarapé da Prata, Rodrigues et al (2016) constataram baixo risco a enchentes e topografia favorável ao escoamento superficial, devido ao seu coeficiente de compacidade apresentar o valor consideravelmente afastado da unidade $(1,829)$ e seu fator de forma 0,356) e índice de circularidade $(0,294)$ apresentarem valores baixos.

A densidade de drenagem da bacia hidrográfica do Rio dos Patos foi igual a 0,78 $\mathrm{km} / \mathrm{km}^{2}$. Beltrame (1994) sugeriu uma classificação em faixas para a densidade de drenagem como mostra a Tabela 2 . 
Tabela 2. Classificação da densidade de drenagem (Dd).

\begin{tabular}{cc}
\hline Dd $\left(\mathbf{k m} / \mathbf{k m}^{\mathbf{2}}\right)$ & Denominação \\
\hline$<0,50$ & Baixa \\
$0,50-2,00$ & Mediana \\
$2,01-3,50$ & Alta \\
$>3,50$ & Muito alta \\
\hline
\end{tabular}

É possível observar a partir da classificação de Beltrame (1994) que a densidade de drenagem da bacia em questão indica que a forma da bacia hidrográfica possui mediana capacidade de drenagem, o que pode estar associado às características do relevo em que o local está inserido, influenciando de maneira significativa na eficiência da drenagem da água.

Ribeiro et al., (2015) caracterizaram a bacia do Igarapé do Una - MG também apresentou faixa de densidade de drenagem mediana segundo a classificação de Beltrame (1994) por apresentar valor de $0,94 \mathrm{Km} / \mathrm{Km}^{2}$ o que é reflexo de um relevo plano, com alta permeabilidade do solo, que permite rapidez de infiltração de água.

Diferente da bacia em estudo Oliveira et al (2010) ao analisar a rede de drenagem da bacia hidrográfica do Ribeirão Salobra - MS, obtiveram valor de $0,30 \mathrm{Km} / \mathrm{Km}^{2}$ o que apresentou baixa densidade de drenagem, porém indica área permeável e de relevo plano e suave.

$\mathrm{Na}$ bacia hidrográfica do Rio Debossan, Nova Friburgo - RJ, a densidade de drenagem encontrada foi de $2,35 \mathrm{~km} / \mathrm{km}^{2}$, apresentando alta densidade de drenagem devida profunda dissecação fluvial e perenidade em função da pluviosidade elevada (CARDOSO et al., 2006).

O coeficiente de compacidade $(\mathrm{Kc})$ de 1,61 e fator de forma (F) 0,22 caracterizou a bacia hidrográfica do Rio dos Patos como alongada e sem grandes riscos de enchentes, pois quanto mais próximo da unidade $(K=1)$ for este coeficiente, mais a bacia se assemelha a um círculo (Tabela 3 e $4)$.

Tabela 3. Classificação do coeficiente de compacidade (Kc).

\begin{tabular}{cc}
\hline Coeficiente de compacidade $(\mathbf{K c})$ & Denominação \\
\hline $1,00-1,25$ & Alta propensão a grandes enchentes \\
$1,25-1,50$ & Mediana a grandes enchentes \\
$>1,50$ & Não sujeita a grandes enchentes \\
\hline
\end{tabular}

Tonello et al., (2006) calcularam o parâmetro do coeficiente de compacidade da bacia hidrográfica da Cachoeira das Pombas, localizada no Município de Guanhães - MG, com isso a bacia em estudo houve semelhança com esta, pelo fato de o coeficiente de compacidade apresentar o valor afastado da unidade (1,575), apresentar o valor tendo uma indicação de que a bacia não possui forma circular, com uma tendência de forma alongada. 
Fato semelhante ocorreu com Salles (2010) ao fazer análise morfométrica da bacia hidrográfica do Rio São João - MG, o coeficiente de compacidade foi de 1,72, valor afastado da unidade e mostra-se não sujeita a grandes enchentes.

Santos et al (2012) analisaram a morfometria das sub-bacias hidrográficas Perdizes e Fojo, Campos do Jordão -SP, e a bacia em estudo t semelhança com ambas, já que respectivamente os fatores de forma foram $\mathrm{F}=0,27$ e $\mathrm{F}=0,29$, e indicaram que estas sub-bacias, não são suscetíveis a enchentes, devido não possuir forma circular.

Tabela 4. Classificação do Fator de Forma (F).

\begin{tabular}{cc}
\hline Fator de forma $(\mathbf{F})$ & Denominação \\
\hline$<0,50$ & Não sujeito a enchentes \\
$0,50-0,75$ & Tendência mediana a enchentes \\
$0,75-1,00$ & Sujeita a enchentes \\
\hline
\end{tabular}

Para corroborar com os resultados do Kc e F, o índice de circularidade $(\mathrm{Ke})$ encontrado foi de 0,38, enfatizando que a bacia hidrográfica do Rio dos Patos possui forma alongada e facilita o processo de escoamento da água. Segundo Schumm (1956), índice de circularidade maior que 0,51 mostra que a bacia tende a ser mais circular, favorecendo os processos de inundação, e valores menores que 0,51 sugerem que a bacia tende a ser mais alongada, o que contribui para o processo de escoamento.

Reis et al., (2017) apresentaram uma caracterização morfométrica da bacia do Rio Guaxindiba, São Francisco de Itabopoama - RJ, onde o índice de circularidade foi de 0,1299, podendo apresentar forma alongada, contribuindo para o processo de escoamento, fato semelhante ocorreu com a bacia em estudo.

Por fim, realizando o resultado da ordem dos cursos de água, a bacia em estudo foi classificada como de $6^{\circ}$ ordem. Conforme Tucci (2001), a ordem dos cursos d'água representa o grau de ramificação do sistema de drenagem da bacia, o que torna a bacia hidrográfica do Rio dos Patos GO, muito ramificada reforçando sua boa drenagem.

Rodrigues et al., (2016) ao caracterizar a bacia hidrográfica de Igarapé da Prata - MG, teve resultado da ordem dos cursos de água, onde a bacia foi classificada $3^{\circ}$ ordem, indicando que o sistema de drenagem da bacia é pouco ramificado.

\section{CONCLUSÕES}

As variáveis morfométricas analisadas indicam que a bacia hidrográfica do rio dos patos possui forma mais alongada, o que a torna pouco susceptível a enchentes e inundações em condições normais de precipitações.

A densidade de drenagem indica que a bacia em estudo possui mediana capacidade de drenagem e possui sistema de drenagem bem ramificado.

A caracterização morfométrica da bacia hidrográfica do Rio dos Patos, GO permite o 
melhor gerenciamento e aproveitamento dos recursos naturais relacionados aos recursos hídricos.

\section{REFERÊNCIAS BIBLIOGRÁFICAS}

ALVES, W. S.; SCOPEL, I.; MARTINS, A. P.; MORAIS, W. A. Análise morfométrica da bacia do Ribeirão das Abóboras - Rio Verde (GO). Geociências, v. 35, n. 4, p.652-667, 2016.

BARRELLA, W. et al As relações entre as matas ciliares os rios e os peixes. In:

RODRIGUES, R.R.; LEITÃO FILHO; H.F. (Ed.) Matas ciliares: conservação e recuperação. 2.ed. São Paulo: Editora da Universidade de São Paulo, 2001.

\section{BELTRAME, A. V. Diagnóstico do meio} ambiente físico de bacias hidrográficas: modelo de aplicação. Florianópolis: UFSC, 1994. 112 p.

BENTES-GAMA, M. de M. Manejo de Bacias Hidrográficas. Informe Técnico. EMBRAPA. Disponível em: < http://www.cpafro.embrapa.br/embrapa/ Artigos/manejo_bac.htm $>$. Acesso em: 22/09/2017.

BRASIL, E. M; CARVALHO, Y. Comportamento de híbridos de milho em relação a Phaeoshaeria maydis em diferentes épocas de plantio. Pesq. Agropec. Bras, v33, p. 1997-1981, 1998.

CARDOSO. C.A.; DIAS. H.C.T.; SOARES. C.P.B.; MARTINS. S.V. Caracterização morfométrica da bacia hidrográfica do Rio Debossan, Nova Friburgo, RJ. Revista Árvore, Viçosa-MG, v.30, n.2, p.241-248, 2006.

CHRISTOFOLETTTI, A. Geomorfologia. 2. ed. São Paulo: Edgard Blucher, 1980. 188 p.

FAUSTINO, J. Planificación y gestión de manejo de cuencas. Turrialba: CATIE, 1996.
GARBRECHT, J.; MARTZ, L.W. Digital elevation model issues in water resources modeling. ESRI, USERS CONFERENCE, 19., 1999, San Diego. Proceedings... San Diego: 1999. CD-ROM.

GOBBI, A. F.; Torres, J. L. R.; Fabian, A. J. Diagnóstico ambiental da microbacia do córrego do Melo em Uberaba- MG. Revista Caminhos de Geografia, v.9, p.206-223, 2008.

HORTON, R. Erosional development of streams and their drainage basins: hidrophysical approach to quatitative morphology. New York: Geological Society of American Bulletin, v.56. p. 807-813, 1945.

LIMA, W. P. Princípios de hidrologia florestal para o manejo de bacias hidrográficas. São Paulo: Escola Superior de Agricultura "Luiz de Queiroz", 1986. 242p.

MARTINS, F. B.; ROCHA, J. S. M.; ROBAINA, A. A.; KURTZ, S. M. J. M.; KURTZ, F. C.; GARCIA, S. M.; SANTOS, A. H. O.; DILL, P. R. J.; NOAL, T.M. Zoneamento Ambiental da sub - bacia hidrográfica do Arroio Cadena, Santa Maria (RS). Estudo de caso. Cerne, v.11, n.3, p.315-322, 2005.

MOTTA, P. N. S. D.; GLOAGUEN, T. V.; SANTOS, M. S. T.; FERREIRA, A. T. S.; MOTTA, T. O. Análise morfométrica da bacia hidrográfica do rio subaé, Bahia, Brasil. Ambiencia, v.13, n.2, p.470-485, 2017.

OLIVEIRA. P.T.S.; SOBRINHO. T.A.; STEFFEN. J.L.; RODRIGUES. D.B.B. Caracterização morfométrica de bacias hidrográficas através de dados SRTM. Revista Brasileira de Engenharia Agrícola e Ambiental v.14, n.8, p.819825, 2010.

REIS, V. N.; ALVES, L. A.; ALVES, M. V.; MIRO, J. M. R. Caracterização morfométrica e dinâmica hídrica na bacia hidrográfica do rio Guaxindiba, São francisco de Itabapoana (RJ). In: Simpósio Brasileiro de Geografia Física Aplicada, 17, 
2017. Anais... Campinas: Unicamp, p. 771783, 2017.

RIBEIRO, E. G. P.; FERREIRA, B. M.; MACIEL, M. N. M.; PEREIRA, B. W. F.; SOARES, J. A. C.; Caracterização morfométrica da bacia hidrográfica do igarapé do una por meio de geotecnologias. Enciclopédia Biosfera, Centro Científico Conhecer - Goiânia, v.11 n.21; p. 2.960. 2015.

ROCHA, J. S. M. Manual de projetos ambientais. Santa Maria, Universidade Federal de Santa Maria, 1997. 423p.

RODRIGUES, R. S. S.; FERNANDES, L. L.; CRISPIM, D. L.; VIEIRA, A. S. A.; PESSOA, F. C. L. Caracterização morfométrica da bacia hidrográfica do Igarapé da Prata, Capitão Poço, Pará, Brasil.

Revista Verde de Agroecologia e Desenvolvimento Sustentável, v.11, No 3 , p. 143-150, 2016.

SALLES, M. M.; O uso do sig na análise morfométrica da bacia hidrográfica do rio São João - MG. 29 f. Monografia. 2010. (Especialização) - Universidade Federal de Minas Gerais, Belo Horizonte, 2010.

SANTOS, A. M.; TARGA, M. S.; BATISTA, G. T.; DIAS, N. W. Análise morfométrica das sub-bacias hidrográficas Perdizes e Fojo no município de Campos do Jordão, SP, Brasil. Ambiente e Água, Taubaté, v. 7, n. 3, p. 195-211, 2012.

SCHUMM, S.A. Evolution of drainage systems and slopes in badlands of Perth Amboy.
Geological Society of America Bulletin, n. 67, p. 597- 646, 1956.

SILVEIRA, A.L.L. Ciclo hidrológico e bacia hidrográfica. In: TUCCI, C.E.M. (Org.). Hidrologia: ciência e aplicação. São Paulo: EDUSP, 2001. p 35-51.

SMITH, K. G. Standars for grading texture of erosional topography. Am. J. Sci., v248 p. 655-668, 1950.

TEIXEIRA, R. C.; BRITO, G. H. M.; ANTUNES, A. M.; ROCHA, I. J. F. Avaliação dos modelos digitais de elevação (MDE) derivados de imagens de sensoriamento remoto orbital. Cientific@, v.5, n.2, p.45-51, 2018.

TONELLO, K. C.; Dias, H. C. T.; Souza, A. L.; Alvares, C. A.; Ribeiro, S.; Leite, F. P. Morfometria da Bacia Hidrográfica da Cachoeira das Pombas, Guanhães - MG. Revista Árvore, v.30, n.5, p.849-857, 2006.

TUCCI, C. E. M.; HESPANHOL, I.; CORDEIRO NETTO, O. M. Cenários da gestão da água no Brasil: uma contribuição para a visão mundial da água. Bahia Análise e Dados, Salvador, v. 13, p.357370, 2003. Número especial.

TUCCI, C. E. M. Hidrologia: ciência e aplicação. Porto Alegre, RS: Editora da Universidade UFRGS/Edusp/ABRH, 2001. 943p.

VILLELA, S. M.; MATTOS, A. Hidrologia aplicada. São Paulo: McGraw-Hill do Brasil, 1975. 245p. 